Supporting information

\title{
Synthesis and Properties of Bis(methylthio)Dithienosilole and Its Oxides
}

Kwang-Hoi Lee, Joji Ohshita, * and Atsutaka Kunai*

Department of Applied Chemistry, Graduate School of Engineering, Hiroshima

University, Higashi-Hiroshima 739-8527, Japan

Table 1. Atomic coordinates and $\mathrm{B}_{\mathrm{iso}} / \mathrm{B}_{\mathrm{eq}}$

$\begin{array}{llcll}\text { atom } & \mathrm{x} & \mathrm{y} & \mathrm{z} & \mathrm{B} \text { eq } \\ \mathrm{S}(1) & 0.65810(8) & 0.01648(6) & 0.65445(6) & 3.82(2) \\ \mathrm{S}(2) & 0.96762(8) & 0.16657(6) & 0.48094(5) & 3.77(2) \\ \mathrm{S}(3) & 0.39988(9) & -0.07360(7) & 0.82175(7) & 4.91(2) \\ \mathrm{S}(4) & 1.1970(1) & 0.32195(7) & 0.36667(6) & 5.04(2) \\ \mathrm{Si}(1) & 0.75971(8) & 0.29990(6) & 0.78162(6) & 3.01(2) \\ \mathrm{C}(1) & 0.5317(3) & 0.0228(2) & 0.7791(2) & 3.41(6) \\ \mathrm{C}(2) & 0.5569(3) & 0.1069(2) & 0.8326(2) & 3.38(6) \\ \mathrm{C}(3) & 0.6771(3) & 0.1689(2) & 0.7759(2) & 3.08(6) \\ \mathrm{C}(4) & 0.7380(3) & 0.1305(2) & 0.6761(2) & 2.93(6) \\ \mathrm{C}(5) & 0.8511(3) & 0.1927(2) & 0.6049(2) & 2.94(6) \\ \mathrm{C}(6) & 0.8800(3) & 0.2880(2) & 0.6430(2) & 3.02(6) \\ \mathrm{C}(7) & 0.9980(3) & 0.3388(2) & 0.5693(2) & 3.48(6) \\ \mathrm{C}(8) & 1.0568(3) & 0.2838(2) & 0.4784(2) & 3.64(6) \\ \mathrm{C}(9) & 0.5724(3) & 0.4331(2) & 0.7802(2) & 3.17(6) \\ \mathrm{C}(10) & 0.4396(4) & 0.4517(3) & 0.7056(3) & 5.97(9) \\ \mathrm{C}(11) & 0.2919(4) & 0.5436(3) & 0.7022(4) & 7.7(1) \\ \mathrm{C}(12) & 0.2744(4) & 0.6183(3) & 0.7778(3) & 6.0(1) \\ \mathrm{C}(13) & 0.4024(4) & 0.6045(3) & 0.8509(3) & 5.50(9) \\ \mathrm{C}(14) & 0.5506(3) & 0.5115(2) & 0.8532(2) & 4.07(7) \\ \mathrm{C}(15) & 0.9158(3) & 0.2806(2) & 0.8951(2) & 3.12(6) \\ \mathrm{C}(16) & 0.9533(3) & 0.1832(2) & 0.9771(2) & 4.19(7) \\ \mathrm{C}(17) & 1.0778(4) & 0.1696(3) & 1.0564(3) & 5.29(8) \\ \mathrm{C}(18) & 1.1691(4) & 0.2514(3) & 1.0534(3) & 5.48(9) \\ \mathrm{C}(19) & 1.1361(4) & 0.3487(3) & 0.9725(3) & 5.9(1) \\ \mathrm{C}(20) & 1.0117(4) & 0.3637(3) & 0.8926(3) & 4.83(8) \\ \mathrm{C}(21) & 0.2186(4) & -0.0086(3) & 0.7342(3) & 8.5(1) \\ \mathrm{C}(22) & 1.4010(4) & 0.2735(4) & 0.4320(3) & 8.9(1)\end{array}$

$\mathrm{B}_{\mathrm{eq}}=8 / 3 \pi^{2}\left(\mathrm{U}_{11}(\mathrm{aa} *)^{2}+\mathrm{U}_{22}\left(\mathrm{bb}^{*}\right)^{2}+\mathrm{U}_{33}(\mathrm{cc} *)^{2}+2 \mathrm{U}_{12}\left(\mathrm{aa} * \mathrm{bb}^{*}\right) \cos \pi+\right.$ $\left.2 \mathrm{U}_{13}\left(\mathrm{aa}^{*} \mathrm{cc} *\right) \cos \pi+2 \mathrm{U}_{23}\left(\mathrm{bb}^{*} \mathrm{cc} *\right) \cos \pi\right)$ 
Table 2. Anisotropic Displacement Parameters

$\begin{array}{lllllll}\text { atom } & \mathrm{U} 11 & \mathrm{U} 22 & \mathrm{U} 33 & \mathrm{U} 12 & \mathrm{U} 13 & \mathrm{U} 23 \\ \mathrm{~S}(1) & 0.0514(4) & 0.0418(4) & 0.0581(5) & -0.0175(3) & 0.0026(3) & -0.0177(4) \\ \mathrm{S}(2) & 0.0544(4) & 0.0488(4) & 0.0422(4) & -0.0142(4) & 0.0074(3) & -0.0159(3) \\ \mathrm{S}(3) & 0.0561(5) & 0.0551(5) & 0.0782(6) & -0.0276(4) & -0.0081(4) & 0.0039(4) \\ \mathrm{S}(4) & 0.0658(5) & 0.0725(6) & 0.0488(5) & -0.0216(4) & 0.0130(4) & 0.0003(4) \\ \mathrm{Si}(1) & 0.0416(4) & 0.0360(4) & 0.0385(4) & -0.0106(3) & -0.0008(3) & -0.0102(3) \\ \mathrm{C}(1) & 0.039(1) & 0.038(2) & 0.051(2) & -0.011(1) & -0.004(1) & -0.000(1) \\ \mathrm{C}(2) & 0.041(1) & 0.043(2) & 0.042(2) & -0.009(1) & -0.000(1) & -0.004(1) \\ \mathrm{C}(3) & 0.039(1) & 0.035(1) & 0.043(2) & -0.009(1) & 0.000(1) & -0.008(1) \\ \mathrm{C}(4) & 0.035(1) & 0.032(1) & 0.046(2) & -0.009(1) & -0.003(1) & -0.010(1) \\ \mathrm{C}(5) & 0.036(1) & 0.039(1) & 0.035(1) & -0.005(1) & -0.000(1) & -0.007(1) \\ \mathrm{C}(6) & 0.042(1) & 0.033(1) & 0.040(1) & -0.010(1) & -0.002(1) & -0.008(1) \\ \mathrm{C}(7) & 0.048(2) & 0.036(2) & 0.046(2) & -0.010(1) & -0.001(1) & -0.002(1) \\ \mathrm{C}(8) & 0.050(2) & 0.044(2) & 0.040(2) & -0.012(1) & 0.003(1) & 0.001(1) \\ \mathrm{C}(9) & 0.043(2) & 0.036(1) & 0.043(2) & -0.010(1) & -0.001(1) & -0.011(1) \\ \mathrm{C}(10) & 0.069(2) & 0.053(2) & 0.108(3) & 0.002(2) & -0.031(2) & -0.035(2) \\ \mathrm{C}(11) & 0.061(2) & 0.071(3) & 0.159(4) & 0.004(2) & -0.049(2) & -0.025(3) \\ \mathrm{C}(12) & 0.060(2) & 0.048(2) & 0.102(3) & 0.006(2) & 0.016(2) & -0.000(2) \\ \mathrm{C}(13) & 0.098(3) & 0.044(2) & 0.056(2) & -0.001(2) & 0.018(2) & -0.012(2) \\ \mathrm{C}(14) & 0.063(2) & 0.046(2) & 0.042(2) & -0.006(1) & 0.001(1) & -0.010(1) \\ \mathrm{C}(15) & 0.039(1) & 0.041(2) & 0.040(1) & -0.009(1) & 0.001(1) & -0.011(1) \\ \mathrm{C}(16) & 0.048(2) & 0.050(2) & 0.057(2) & -0.009(1) & -0.002(1) & -0.003(1) \\ \mathrm{C}(17) & 0.059(2) & 0.069(2) & 0.059(2) & -0.001(2) & -0.013(2) & 0.007(2) \\ \mathrm{C}(18) & 0.051(2) & 0.091(3) & 0.065(2) & -0.002(2) & -0.022(2) & -0.024(2) \\ \mathrm{C}(19) & 0.072(2) & 0.072(2) & 0.090(3) & -0.030(2) & -0.026(2) & -0.016(2) \\ \mathrm{C}(20) & 0.067(2) & 0.057(2) & 0.063(2) & -0.022(2) & -0.019(2) & -0.000(2) \\ \mathrm{C}(21) & 0.063(2) & 0.136(3) & 0.125(3) & -0.047(2) & -0.033(2) & 0.018(3) \\ \mathrm{C}(22) & 0.075(2) & 0.181(4) & 0.088(3) & -0.057(3) & 0.005(2) & -0.005(3)\end{array}$

The general temperature factor expression: $\exp \left(-2 \pi^{2}\left(\mathrm{a}^{*} \mathrm{U}_{11} \mathrm{~h}^{2}+\mathrm{b}^{* 2} \mathrm{U} 22 \mathrm{k}^{2}+\right.\right.$ $\left.\left.\mathrm{c}^{*} \mathrm{U}_{33} \mathrm{l}^{2}+2 \mathrm{a} * \mathrm{~b} * \mathrm{U}_{12} \mathrm{hk}+2 \mathrm{a} * \mathrm{c} * \mathrm{U}_{13} \mathrm{hl}+2 \mathrm{~b} * \mathrm{c} * \mathrm{U}_{23} \mathrm{kl}\right)\right)$

Table 3. Bond lengths $(\AA)$

$\begin{array}{llllll}\text { atom } & \text { atom } & \text { distance } & \text { atom } & \text { atom } & \text { distance } \\ \mathrm{S}(1) & \mathrm{C}(1) & 1.743(2) & \mathrm{S}(1) & \mathrm{C}(4) & 1.722(2) \\ \mathrm{S}(2) & \mathrm{C}(5) & 1.729(2) & \mathrm{S}(2) & \mathrm{C}(8) & 1.730(2) \\ \mathrm{S}(3) & \mathrm{C}(1) & 1.742(2) & \mathrm{S}(3) & \mathrm{C}(21) & 1.771(3) \\ \mathrm{S}(4) & \mathrm{C}(8) & 1.752(2) & \mathrm{S}(4) & \mathrm{C}(22) & 1.763(3) \\ \mathrm{Si}(1) & \mathrm{C}(3) & 1.872(2) & \mathrm{Si}(1) & \mathrm{C}(6) & 1.869(2) \\ \mathrm{Si}(1) & \mathrm{C}(9) & 1.870(2) & \mathrm{Si}(1) & \mathrm{C}(15) & 1.862(2) \\ \mathrm{C}(1) & \mathrm{C}(2) & 1.359(3) & \mathrm{C}(2) & \mathrm{C}(3) & 1.420(3) \\ \mathrm{C}(3) & \mathrm{C}(4) & 1.376(3) & \mathrm{C}(4) & \mathrm{C}(5) & 1.448(3) \\ \mathrm{C}(5) & \mathrm{C}(6) & 1.387(3) & \mathrm{C}(6) & \mathrm{C}(7) & 1.418(3) \\ \mathrm{C}(7) & \mathrm{C}(8) & 1.371(3) & \mathrm{C}(9) & \mathrm{C}(10) & 1.386(4)\end{array}$




$\begin{array}{llllll}\mathrm{C}(9) & \mathrm{C}(14) & 1.374(3) & \mathrm{C}(10) & \mathrm{C}(11) & 1.377(4) \\ \mathrm{C}(11) & \mathrm{C}(12) & 1.369(4) & \mathrm{C}(12) & \mathrm{C}(13) & 1.355(4) \\ \mathrm{C}(13) & \mathrm{C}(14) & 1.387(3) & \mathrm{C}(15) & \mathrm{C}(16) & 1.372(3) \\ \mathrm{C}(15) & \mathrm{C}(20) & 1.401(3) & \mathrm{C}(16) & \mathrm{C}(17) & 1.388(4) \\ \mathrm{C}(17) & \mathrm{C}(18) & 1.360(4) & \mathrm{C}(18) & \mathrm{C}(19) & 1.362(4) \\ \mathrm{C}(19) & \mathrm{C}(20) & 1.388(4) & & & \end{array}$

Table 5. Bond angles (O)

$\begin{array}{llllllll}\text { atom } & \text { atom } & \text { atom } & \text { angle } & \text { atom } & \text { atom } & \text { atom } & \text { angle } \\ \mathrm{C}(1) & \mathrm{S}(1) & \mathrm{C}(4) & 91.0(1) & \mathrm{C}(5) & \mathrm{S}(2) & \mathrm{C}(8) & 91.3(1) \\ \mathrm{C}(1) & \mathrm{S}(3) & \mathrm{C}(21) & 101.2(1) & \mathrm{C}(8) & \mathrm{S}(4) & \mathrm{C}(22) & 101.0(2) \\ \mathrm{C}(3) & \mathrm{Si}(1) & \mathrm{C}(6) & 91.4(1) & \mathrm{C}(3) & \mathrm{Si}(1) & \mathrm{C}(9) & 110.3(1) \\ \mathrm{C}(6) & \mathrm{Si}(1) & \mathrm{C}(9) & 114.4(1) & \mathrm{C}(3) & \mathrm{Si}(1) & \mathrm{C}(15) & 115.2(1) \\ \mathrm{C}(6) & \mathrm{Si}(1) & \mathrm{C}(15) & 110.5(1) & \mathrm{C}(9) & \mathrm{Si}(1) & \mathrm{C}(15) & 113.3(1) \\ \mathrm{S}(1) & \mathrm{C}(1) & \mathrm{S}(3) & 120.5(1) & \mathrm{S}(1) & \mathrm{C}(1) & \mathrm{C}(2) & 111.0(2) \\ \mathrm{S}(3) & \mathrm{C}(1) & \mathrm{C}(2) & 128.6(2) & \mathrm{C}(1) & \mathrm{C}(2) & \mathrm{C}(3) & 114.3(2) \\ \mathrm{Si}(1) & \mathrm{C}(3) & \mathrm{C}(2) & 140.7(2) & \mathrm{Si}(1) & \mathrm{C}(3) & \mathrm{C}(4) & 107.8(2) \\ \mathrm{C}(2) & \mathrm{C}(3) & \mathrm{C}(4) & 110.8(2) & \mathrm{S}(1) & \mathrm{C}(4) & \mathrm{C}(3) & 112.9(2) \\ \mathrm{S}(1) & \mathrm{C}(4) & \mathrm{C}(5) & 130.3(2) & \mathrm{C}(3) & \mathrm{C}(4) & \mathrm{C}(5) & 116.7(2) \\ \mathrm{S}(2) & \mathrm{C}(5) & \mathrm{C}(4) & 131.5(2) & \mathrm{S}(2) & \mathrm{C}(5) & \mathrm{C}(6) & 112.3(2) \\ \mathrm{C}(4) & \mathrm{C}(5) & \mathrm{C}(6) & 116.1(2) & \mathrm{Si}(1) & \mathrm{C}(6) & \mathrm{C}(5) & 107.8(2) \\ \mathrm{Si}(1) & \mathrm{C}(6) & \mathrm{C}(7) & 140.9(2) & \mathrm{C}(5) & \mathrm{C}(6) & \mathrm{C}(7) & 111.1(2) \\ \mathrm{C}(6) & \mathrm{C}(7) & \mathrm{C}(8) & 114.0(2) & \mathrm{S}(2) & \mathrm{C}(8) & \mathrm{S}(4) & 120.6(1) \\ \mathrm{S}(2) & \mathrm{C}(8) & \mathrm{C}(7) & 111.3(2) & \mathrm{S}(4) & \mathrm{C}(8) & \mathrm{C}(7) & 128.0(2) \\ \mathrm{Si}(1) & \mathrm{C}(9) & \mathrm{C}(10) & 118.7(2) & \mathrm{Si}(1) & \mathrm{C}(9) & \mathrm{C}(14) & 124.5(2) \\ \mathrm{C}(10) & \mathrm{C}(9) & \mathrm{C}(14) & 116.7(2) & \mathrm{C}(9) & \mathrm{C}(10) & \mathrm{C}(11) & 123.1(3) \\ \mathrm{C}(10) & \mathrm{C}(11) & \mathrm{C}(12) & 118.2(3) & \mathrm{C}(11) & \mathrm{C}(12) & \mathrm{C}(13) & 120.7(3) \\ \mathrm{C}(12) & \mathrm{C}(13) & \mathrm{C}(14) & 120.4(3) & \mathrm{C}(9) & \mathrm{C}(14) & \mathrm{C}(13) & 121.0(3) \\ \mathrm{Si}(1) & \mathrm{C}(15) & \mathrm{C}(16) & 123.0(2) & \mathrm{Si}(1) & \mathrm{C}(15) & \mathrm{C}(20) & 119.4(2) \\ \mathrm{C}(16) & \mathrm{C}(15) & \mathrm{C}(20) & 117.4(2) & \mathrm{C}(15) & \mathrm{C}(16) & \mathrm{C}(17) & 121.0(3) \\ \mathrm{C}(16) & \mathrm{C}(17) & \mathrm{C}(18) & 120.9(3) & \mathrm{C}(17) & \mathrm{C}(18) & \mathrm{C}(19) & 119.5(3) \\ \mathrm{C}(18) & \mathrm{C}(19) & \mathrm{C}(20) & 120.3(3) & \mathrm{C}(15) & \mathrm{C}(20) & \mathrm{C}(19) & 120.8(3)\end{array}$




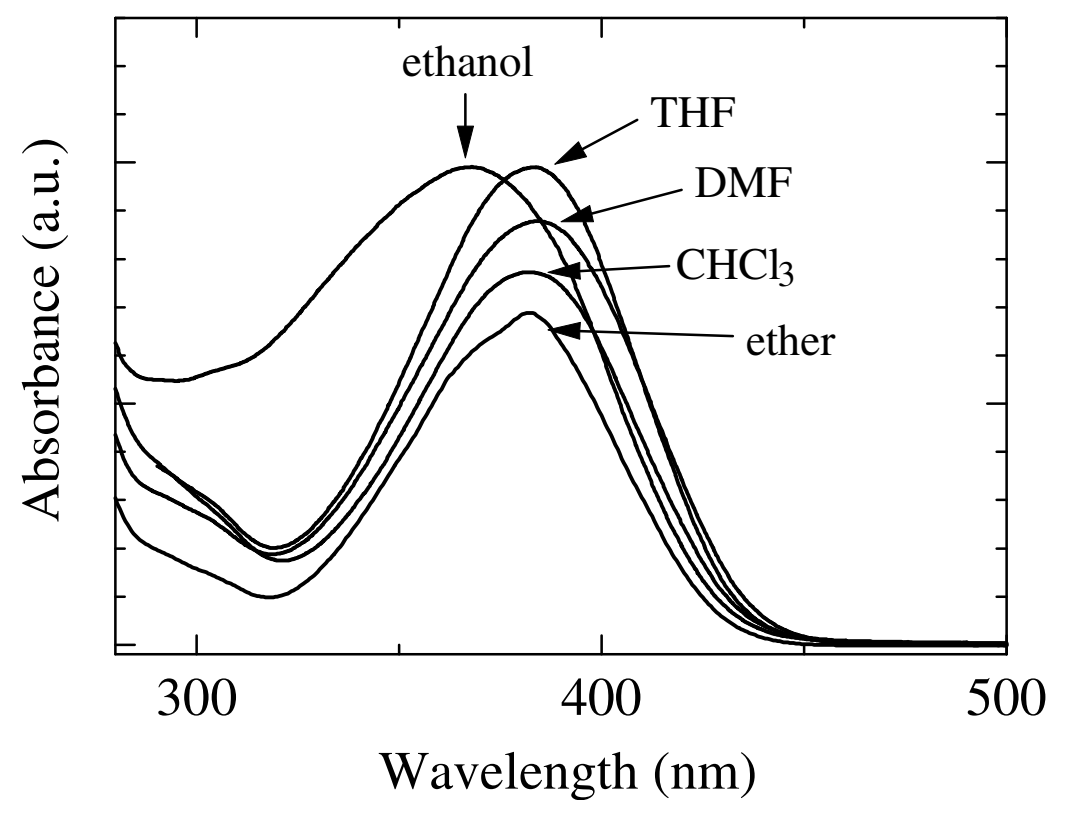

Figure 1. UV spectra of compound 2 in ethanol, THF, DMF, $\mathrm{CHCl}_{3}$, and ether.

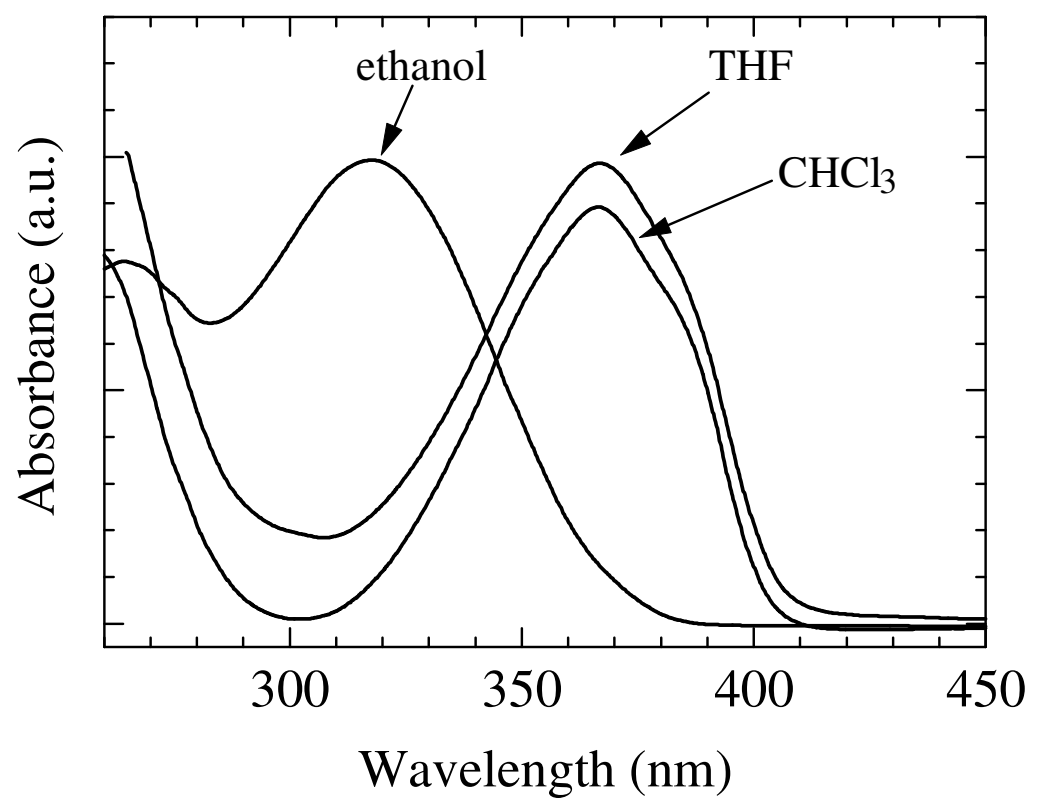

Figure 2. UV spectra of compound 3 in ethanol, THF, and $\mathrm{CHCl}_{3}$. 


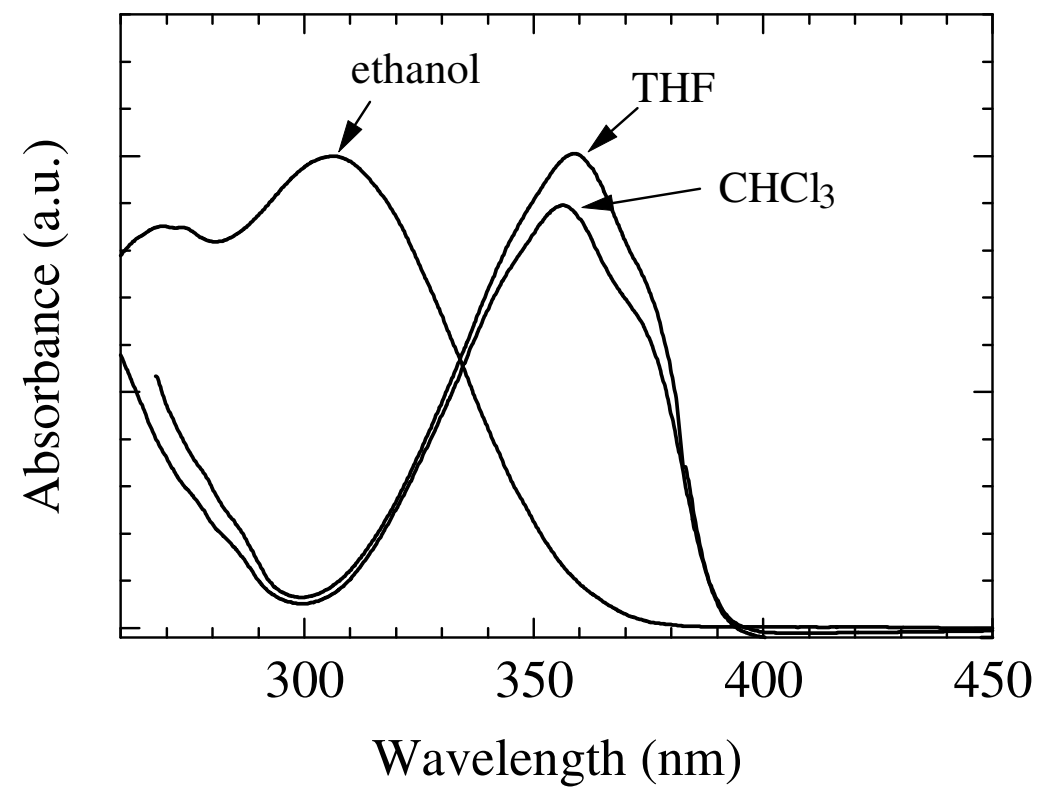

Figure 3. UV spectra of compound 4 in ethanol, $\mathrm{THF}$, and $\mathrm{CHCl}_{3}$. 